\title{
Substrate Binding to Cytochrome P450-2J2 in Nanodiscs Detected by Nanoplasmonic Lycurgus Cup Arrays
}

Lisa Plucinski ${ }^{\dagger \ddagger}$, Manas Ranjan Gartia ${ }^{\dagger \ddagger}$, William R. Arnold ${ }^{\ddagger}$, Abid Ameen ${ }^{\square}$ Te-Wei Chang ${ }^{\dagger}$, Austin Hsiao ${ }^{\square}$, Gang Logan $\operatorname{Liu}^{\dagger *}$, and Aditi Das $\stackrel{\perp_{¥} \S^{*}}{ }$

${ }^{\dagger}$ Department of Electrical and Computer Engineering, Micro and Nanotechnology Laboratory, University of Illinois at Urbana-Champaign, 208 North Wright Street, Urbana, Illinois, 61801, United States

${ }^{\bar{T}}$ Department of Materials Science and Engineering, Micro and Nanotechnology Laboratory, University of Illinois at Urbana-Champaign, 208 North Wright Street, Urbana, Illinois, 61801, United States

${ }^{\ddagger}$ Department of Biochemistry, University of Illinois at Urbana-Champaign, 208 North Wright Street, Urbana, Illinois, 61801, United States

Department of Bioengineering, Micro and Nanotechnology Laboratory, University of Illinois at Urbana-Champaign, 208 North Wright Street, Urbana, Illinois, 61801, United States

${ }^{\perp}$ Department of Comparative Biosciences, University of Illinois at Urbana-Champaign, 208

North Wright Street, Urbana, Illinois, 61801, United States

${ }^{\S}$ Beckman Institute for Advanced Science and Technology and Department of Bioengineering, University of Illinois at Urbana-Champaign, 208 North Wright Street, Urbana, Illinois, 61801,

United States

* These authors contributed equally

*Corresponding Authors: aditidas@illinois.edu (Aditi Das), loganliu @illinois.edu (Gang Logan Liu) 


\begin{abstract}
Cytochrome P450s are the primary enzymes involved in phase I drug metabolism. They are an important target for early drug discovery research. However, high-throughput drug screening of P450s is limited by poor protein stability and lack of consistent measurement of binding events. Here we present the detection of substrate binding to cytochrome P450-2J2 (CYP2J2), the predominant P450 in the human heart, using a combination of Nanodisc technology and a nanohole plasmonic sensor called nanoplasmonic Lycurgus cup array (nanoLCA). The Nanodisc, a nanoscale membrane bilayer disc, is used to stabilize the protein on the metallic plasmonic surface. Absorption spectroscopy of seven different substrates binding to CYP2J2 in solution showed that they are all type I, resulting in shifting of the protein bands to lower wavelengths (blue shift). Detection on the nanoLCA sensor also showed spectral blue shifts of CYP2J2 following substrate binding. Finite Difference Time Domain (FDTD) electromagnetic simulation suggested that the blue shift on the nanoLCA is because of the hybridization of plasmon polariton Bloch wave and the electronic resonance of the heme group of CYP2J2. We found the plasmonic properties of the nanoLCA sensor to be highly reproducible, which allowed comparisons among the different substrates at different concentrations. Further, due to the unique spectral properties of the nanoLCA sensor, including the transmission of a single color, we were able to perform colorimetric detection of the binding events. These results indicate that a resonance plasmonic sensing mechanism can be used to distinguish between different substrates of the same binding type at different concentrations binding to P450s and that the nanoLCA sensor has the potential to provide consistent high-throughput measurements of this system.
\end{abstract}




\section{Introduction}

The development of a high-throughput label-free method for detecting substrate binding to cytochrome P450s would be an important advance in early stage drug discovery research. In addition to their crucial roles in xenobiotic metabolism, P450s are responsible for important drug-drug interactions which can potentially lead to drug toxicity and fluctuations in protein enzymatic activity (Guengerich 1999, 2008; Rittle and Green 2010; Whitlock and Denison 1995). Several challenges limit the ability to screen drug binding to P450s including that the proper expression, isolation, and purification of these proteins at a high yield is labor intensive (Gillam et al. 1993).

Currently drug binding to P450s is detected using absorbance spectroscopic assays in solution (Luthra et al. 2011). Detection is based on the fact that after drug binding P450s can shift the absorbance peak at 417nm (Soret band) to either shorter wavelengths (blue shift, type I binding) or to higher wavelengths (red shift, type II binding) (Davydov and Halpert 2008; Isin and Guengerich 2006, 2008; Wells et al. 1992). Label-free optical devices, such as the photonic crystal (Ganesh et al. 2007), ring resonator (Iqbal et al. 2010), interferometer (Lin et al. 1997), and surface plasmon resonance (SPR) sensor (Piliarik et al. 2009; Rich and Myszka 2000) are especially promising for surface-based drug screening applications, which increase sensitivity and reduce the required sample amount. However, these techniques remain limited by the complex and bulky external optical systems required for signal generation, detection, and analysis.

In addition to SPR, plasmonic biosensing can be accomplished by localized surface plasmon resonance (LSPR) and extraordinary optical transmission (EOT). In LSPR, the electric field near metal nanoparticles is amplified resulting in strong scattering spectra ideal for 
applications in protein biosensing (Anker et al. 2008; Baciu et al. 2008; Wu et al. 2012). Unlike SPR, which uses a metallic thin film and prism system, LSPR does not require a complex system in order to couple the light source to the metal dielectric interface (Haes and Van Duyne 2004). However, the difficulty in fabricating uniform sensors over a large surface area leads to reduced reliability and limited applications. EOT sensors consist of periodic arrays of nanoholes in metallic thin films (Gordon et al. 2008). Transmission through these films is orders of magnitude greater than that predicted by classical aperture theory partially due to the excitation of surface plasmon resonances (Escobedo 2013). This reliance on SPR has enabled applications including protein binding (Ji et al. 2009), exosome profiling (Im et al. 2014), and virus detection (Yanik et al. 2010). Similar to LSPR, these sensors do not require a complex optical setup, but it is difficult to fabricate uniform devices over a large surface area.

If there is an overlap between the LSPR device resonance and the molecular resonance of a colored adsorbate, then resonance LSPR can result in larger shifts (Haes et al. 2006). Resonance LSPR has been used to detect drug binding to P450s by measuring the spectral shift of the P450 protein after small molecule binding (Zhao et al. 2008; Zhao et al. 2006). The ability to distinguish between type I and type II substrates has been demonstrated using silver LSPR sensors fabricated by nanosphere lithography (Das et al. 2009). The further development of LSPR detection of substrate binding to P450s requires wafer-scale nanoplasmonic sensors with minimum defects and therefore consistent spectral properties. In addition, while silver nanoparticles have a higher sensitivity, gold nanoparticles are inert, making them more compatible with high-throughput drug screening (Karlsson and Stahlberg 1995; Zeng et al. 2011). 
Herein we report the application of a gold nanoplasmonic Lycurgus cup array (nanoLCA) sensor to the detection of substrate binding to P450s. The tapered nanohole shape of the nanoLCA has a metal layer $(90 \mathrm{~nm})$ at the rim of the holes and at the bottom of the holes (90 $\mathrm{nm}$ ), providing an intense electromagnetic field at the bottom and top of the nanohole due to LSPR (Gartia et al. 2013). In addition, during deposition, gold nanoparticles form on the tapered cup sidewalls, contributing to LSPR. The periodicity of nanohole structures also provide surface plasmon polariton-Bloch wave (SPP-BW) due to lattice plasmons. The nanoLCA sensor then operates based on a distinct combination of SPP-BW and LSPR plasmonic properties. Due to the high intensity of LSPR by nanoparticle scattering, the nanoLCA transmission spectrum is characterized by a single peak in the entire visible range.

Crucial parameters characterizing the nanoLCA including the deposited metal layer thickness, height, and pitch have been previously optimized to maximize the sensitivity and minimize the full width half maximum (FWHM) of the transmitted resonance peak (Gartia et al. 2013). We have previously reported the sensitivity of the gold nanoLCA to be $247 \mathrm{~nm} \mathrm{RIU}^{-1}$, the figure of merit (FOM) to be 3.3 with a FWHM of $75 \mathrm{~nm}$, and the limit of detection (LOD) to be $7.98 \times 10^{-5}$ RIU. Here sensitivity is defined as $\left(\frac{\Delta \lambda}{\Delta n}\right)$, FOM is defined as $\left(\frac{\Delta \lambda}{\Delta n}\right)\left(\frac{1}{\Delta w}\right)$, and LOD is defined as $\left(\frac{2 \sigma \Delta n}{\Delta \lambda}\right)$ where $\Delta \lambda$ is the shift in the plasmon resonance peak, $\Delta n$ is the change in the refractive index, $\Delta w$ is the FWHM of the plasmon resonance peak, and $\sigma$ is the standard deviation of noise (0.00986 nm) (Gartia et al. 2013; Hsiao et al. 2015).

While the nanoLCA can be applied to measure drug binding to any P450, here the detection of substrate binding to cytochrome P450-2J2 (CYP2J2) is demonstrated. CYP2J2 is the most common P450 found in the human heart and its primary role is the metabolism of arachidonic acid (AA) (Delozier et al. 2007; Wu et al. 1996). This produces epoxyeicosatrienoic 
acids, which are required for proper cardiovascular function (Spector et al. 2004; Zeldin 2001).

Additionally, CYP2J2 metabolizes drugs, many of which have been shown to be cardiotoxic. Therefore, it is of interest to know what xenobiotics bind to CYP2J2 in order to assess their interference to AA metabolism.

CYP2J2 is found in heart and is involved in the metabolism of endogenous fatty acids such as arachidonic acid (AA) and 2-arachidonyl glycerol (2-AG). Therefore these were two obvious substrates to study (McDougle et al. 2014; McDougle et al. 2013). CYP2J2 is also known to metabolize several drugs and the predominant drug were chosen for this study (terfenadine, TFN; ebastine, EBA; MSPPOH; danazol, DAN; and doxorubicin, DOX) (Hashizume et al. 2002; Lafite et al. 2007; Lee et al. 2010; McDougle et al. 2014; Zhang et al. 2009).

Small molecule binding to CYPs is typically assessed using spectral shift titrations (Zhao et al. 2008; Zhao et al. 2006). Typically we measure the shift of the Soret at $417 \mathrm{~nm}$ to higher or lower wavelength. The extent of this shift is dependent on the substrate. We determined the dissociation constants of each substrate from these shifts as explained in the manuscript. We could not do similar studies with DOX as there is severe spectral overlap between DOX and heme absorbance. We show that we can overcome such difficulties using nanoLCA sensor.

Most eukaryotic P450s are membrane-bound and outside the cellular environment they tend to denature and become unstable both in solution and when immobilized on surfaces (Bayburt and Sligar 2002, 2003; Sligar 2003). In order to circumvent this challenge, we use Nanodiscs to stabilize CYP2J2. Nanodiscs consist of nanosized soluble lipid bilayers held together by membrane scaffold proteins (Denisov et al. 2004; Nath et al. 2007). The use of Nanodiscs is critical for this study because they ensure that the CYP2J2 will be immobilized on the nanoLCA sensor surface in an active conformation. 
We report the measurement of the spectral change of immobilized CYP2J2-Nanodiscs (CYP2J2-NDs) corresponding to the binding of seven different type I substrates at two different concentrations and the comparison of these results with conventional solution-based absorption spectroscopy. The resonance of the nanoLCA sensor occurs in the visible range, allowing detection by a conventional halogen light source and portable spectrometer. We also report the spectral reliability of the nanoLCA sensor, which allows for the assessment of substratedependent and concentration-dependent shifts. Finally, we introduce the use of bright-field microscope images in order to detect substrate binding to CYP2J2-NDs on the nanoLCA device.

\section{Materials and Methods}

2.1 Materials. Human CYP2J2 cDNA was obtained from OriGene (Catalog No. SC321730) and modified (Rich and Myszka 2000). Ampicillin, arabinose, chloramphenicol, IPTG and Ni-NTA resin were obtained from Gold Biotechnology and Sigma. $\delta$-aminolevulinic acid was bought from Frontier Scientific. 1-palmitoyl-2-oleoyl-sn-glycero-3-phosphocholine (POPC) and 1hexadecanoyl-2-(9Z-octadecenoyl)-sn-glycero-3-phospho-L serine (POPS) were purchased from Avanti Polar Lipids, Inc. AA, 2-AG, and MSPPOH were purchased from Cayman Chemical. Other drugs were purchased from the following companies: MP Biomedical (Danazol), Santa Cruz Biotechnology (Ebastine), and Gold Biotech (Doxorubicin). UV-curable polymer was purchased from Norland Products (NOA-61). All other materials and reagents used were purchased from Sigma and Fisher Scientific.

2.2 Recombinant Expression of CYP2J2 in E. coli. CYP2J2-D34 was expressed and purified according to previous methods (McDougle et al. 2013; Zelasko et al. 2013). Briefly, a starter culture of DH5 $\alpha$ E. coli cells containing CYP2J2 and pTGro7 chaperonin plasmids were grown 
in $25 \mathrm{~mL}$ of Luria Bertani (LB) media containing the antibiotics chloroamphenicol $(20 \mu \mathrm{g} / \mathrm{mL})$ and ampicillin $(100 \mu \mathrm{g} / \mathrm{mL})$ at $37^{\circ} \mathrm{C}$ and $220 \mathrm{rpm}$ overnight. This culture was then used to inoculate 6 x $500 \mathrm{~mL}$ of Terrific Broth (TB) containing chloroamphenicol $(20 \mu \mathrm{g} / \mathrm{mL})$ and ampicillin $(100 \mu \mathrm{g} / \mathrm{mL})$. The culture was grown for 2.5 hours at $37^{\circ} \mathrm{C}$ and $220 \mathrm{rpm} .0 .1 \mathrm{mM} \delta$ aminolevulinic acid was added and the culture was grown at $26^{\circ} \mathrm{C}$ and $160 \mathrm{rpm}$ until $\mathrm{OD}_{600}=1.0$ was reached. Cells were induced with $1 \mathrm{mM}$ Isopropyl $\beta$-D-1-thiogalactopyranoside (IPTG) and 2 $\mathrm{g}$ of arabinose and left to grow for 44 hours. The cells were subsequently centrifuged at 8,000 rpm and $4^{\circ} \mathrm{C}$ for 15 min. using a Sorvall GSA (Thermo Scientific, Pittsburg PA) rotor. The cells were resuspended in a lysis buffer containing $0.1 \mathrm{mM}$ DTT, $0.2 \mathrm{mM}$ phenylmethanesulfonylfluoride (PMSF), and $5 \mathrm{mg}$ DNase, and then lysed via sonication (5 x 30 sec on/off cycles). The membrane fraction was isolated using ultracentrifugation at 35,000 rpm and $4^{\circ} \mathrm{C}$ for 30 min. with a Ti-45 rotor (Beckman Coulter, Brea, CA). The pellet was resuspended in $0.1 \mathrm{M}$ potassium phosphate buffer $(\mathrm{KPi})$ containing $1.0 \%(\mathrm{w} / \mathrm{v})$ sodium cholate, $20 \%$ glycerol, and $0.2 \mathrm{M} \mathrm{NaCl}$ (column buffer) at $4^{\circ} \mathrm{C}$ for four hours with stirring in order to extract the protein. The insoluble fraction was removed via a second ultracentrifugation at $35,000 \mathrm{rpm}$ and $4^{\circ} \mathrm{C}$ for $30 \mathrm{~min}$. and the supernatant was applied to a Ni-NTA column to purify His-tagged CYP2J2. Protein was eluted using column buffer containing 200mM imidazole. The yield of the protein was $\sim 200 \mathrm{nmol} / \mathrm{L}$.

2.3 Incorporation of CYP2J2 into Nanodiscs. Nanodiscs were prepared as previously described (McDougle et al. 2013). A lipid mixture of 20:80 POPS:POPC in $\mathrm{CHCl}_{3}$ was dried under a flow of $\mathrm{N}_{2}$ gas. The dried lipids were then reconstituted in buffer containing $0.1 \mathrm{M} \mathrm{KPi}$ (pH 7.4) and 200mM cholate. Membrane scaffold protein MSP1E3D1 was added (1:130 MSP:lipids) and allowed to incubate for half an hour at $4^{\circ} \mathrm{C}$. Purified CYP2J2 was then added 
(0.1M Kpi (pH 7.4), 20\% glycerol, and 0.1\% cholate) in a 1:10 CYP2J2:MSP ratio and incubated for one more hour before the addition of Amberlite beads to remove the detergent. This mixture was incubated overnight. Discs were purified via size-exclusion chromatography using an Alliance 2695 analytical separation module (Waters, Milford, MA) coupled to a Waters 996 photodiode array detector (Waters) and a Superdex 10/200 column (GE Healthcare).

2.4 Drug Binding to CYP2J2-Nanodiscs in Solution. Substrate binding was determined by monitoring the Soret shift from 420-390 nm. Absorbance spectra were taken using a Cary Bio 300 UV-Vis spectrophotometer (Agilent Technologies, Santa Clara, CA). An initial spectrum of CYP2J2-Nanodiscs in 0.1M KPi (pH 7.4) was taken at $37^{\circ} \mathrm{C}$. Substrates were then titrated into this cuvette to saturating amounts. Total volume of organics remained $<2 \%$ of the initial volume. To correct for the absorbance of the substrates, separate titration experiments were performed using empty Nanodiscs (without CYP2J2), and these readings were subtracted from the CYP2J2Nanodisc spectra.

2.5 NanoLCA Fabrication and Characterization. The tapered nanohole array was made using a previously described method (Gartia et al. 2013). Briefly, the plasmonic structures were prepared using a replica molding process. The mold consisting of a two dimensional square array of nanocup structures with a lattice constant of $\sim 350 \mathrm{~nm}$ was first prepared on a glass substrate using laser interference lithography. The two-dimensional square array was transferred to a flexible and optically transparent polyethylene terephthalate (PET) film by nanoreplica molding. To prepare the master substrate for transfer, the mold was cleaned and silanized (Repel-silane ES GE Healthcare, Sigma) for $30 \mathrm{~min}$. followed by ethanol and DI water rinse. A $2 \mathrm{~mL}$ drop of UVcurable polymer (NOA-61) was evenly spread on the top of the nanocone master and a supporting PET sheet was carefully put on top of the polymer. The master with the polymer and 
PET sheet was then exposed to UV-light $\left(105 \mathrm{~mW} \mathrm{~cm}^{-2}\right)$ for $60 \mathrm{sec}$. After curing, the complimentary nanohole structures were transferred onto the polymer, which was peeled off carefully from the master mold to complete the transfer process. In order to make the device surface plasmon active, $90 \mathrm{~nm}$ of gold along with a $5 \mathrm{~nm}$ titanium adhesive layer was deposited using electron beam deposition (Temescal six pocket E-Beam Evaporator).

\subsection{Immobilization of CYP2J2-Nanodiscs on NanoLCA and Spectral Measurements. A}

square device with a $2 \mathrm{~cm}$ side length was cut from the prepared nanoLCA and it was cleaned with isopropanol (IPA) and DI water followed by rinsing with IPA and drying with $\mathrm{N}_{2}$. The sensor was then treated with oxygen plasma (PICO plasma cleaner) for $60 \mathrm{sec}$. and four separate circular wells were formed on the surface using PDMS applied with UV curable polymer (NOA61). Each well held $45 \mu \mathrm{L}$ of solution. To being CYP2J2-ND immobilization, each well was filled with a 10mM 11-MUA solution in 70\% ethanol and incubation was done at room temperature for 24 hours. After immersion, each well was washed three times in $70 \%$ ethanol followed by drying with $\mathrm{N}_{2}$. CYP2J2-ND immobilization to the MUA monolayer occurred by EDC activation. Each well was filled with $10 \mathrm{mM}$ EDC and $8 \mu \mathrm{M}$ CYP2J2-ND in 10mM potassium phosphate (KPi) buffer. The EDC and protein were mixed immediately before pipetting into the wells in order to minimize EDC self-reaction and CYP2J2-ND cross-linking. The sensor was incubated in the CYP2J2-ND solution for 1 hour at $37^{\circ} \mathrm{C}$. After incubation, each well was rinsed three times with DI water and dried with $\mathrm{N}_{2}$. Illumination and detection of binding events was accomplished with an upright microscope (Olympus BX51, PA, USA) with a halogen light source using a 20X objective and a portable spectrometer (Ocean Optics, Dunedin, FL). Successful immobilization of MUA and CYP2J2-ND was monitored by measuring the red shift in the transmission spectrum for each well of each device. 
2.7 Substrate Binding to CYP2J2-Nanodiscs on NanoLCA. A device containing four PDMS wells was used for a single substrate. Two of the wells were replicates used for a high concentration of the substrate, one well was used for a low concentration of the substrate, and the fourth well was used for the vehicle control, which was $10 \mathrm{mM}$ KPi buffer. The seven substrates tested were the fatty acids AA and 2-Arachidonoylglycerol (2-AG), and the drugs doxorubicin (DOX), ebastine, MSPPOH, terfenadine (TFN), and danazol (DAN). Incubation was done for 30 minutes at $37^{\circ} \mathrm{C}$. The dissociation constants for all drugs with CYP2J2-ND were measured using solution-based titrations and the maximum value was $20 \mu \mathrm{M}$. For the high concentration $300 \mu \mathrm{M}$ was used and for the low concentration we used a value that was half of the measured dissociation constant for each substrate. After incubation, each well was rinsed with DI water three times and dried with $\mathrm{N}_{2}$ before taking the transmission spectrum.

2.8 Data Analysis. The light source transmission spectrum was measured after each binding step. Every transmission spectrum was then normalized with the light source spectrum for that step in order to obtain the transmissivity and was then smoothened by the Loess Method using OriginPro 9.1 (Origin Labs Inc., Northhampton, MA). It is expected that with a binding event there will be a corresponding shift in the plasmon resonance peak as well as a change in the peak intensity. Here the focus is on the spectral shift and not the intensity change; therefore all transmissivity data was normalized to one in order to better visualize the peak shifts. A Gaussian fit was then performed on each peak in order to obtain the plasmon resonance peak wavelength for each step using OriginPro 9.1 (Origin Labs Inc., Northhampton, MA). The solution-based absorbance data was processed using Matlab R2014a (Mathlab Inc., MI) by subtracting the substrate-free spectrum from each titration spectra. The resulting peak and trough differences 
were then plotted and fitted to the Michaelis-Menten equation using OriginPro 9.1 (Origin Labs Inc., Northhampton, MA).

2.9 FDTD Simulation. 3D-FDTD (finite difference time domain) electromagnetic numerical computation was performed by using the FDTD software package from Lumerical Solutions. The total simulation region was set to $350 \mathrm{~nm} \times 350 \mathrm{~nm} \times 800 \mathrm{~nm}$. The boundary conditions in $\mathrm{x}$ and y directions were set to be periodic to present the array effect of nanoLCA device. The perfect matching layer (PML) was applied in $\mathrm{z}$-directional boundary condition to minimize the simulation error from boundary reflection. The $\mathrm{x}$-axis polarized plane wave was set to propagate normal to the substrate (-z direction). Modeling of the nanostructure was based on dimensions measured by SEM images of the nanoLCA device. The mesh size was set to be $5 \mathrm{~nm}$ in order to minimize stair-case and dispersion errors.

\section{Results and Discussion}

3.1 Substrate Binding to CYP2J2-Nanodiscs. Detection of substrate binding to CYP2J2-NDs on the nanoLCA sensor required immobilization of CYP2J2-NDs with the active site of CYP2J2 available. Figure 1(A) shows a schematic of an immobilized CYP2J2-ND on the nanoLCA and Figure 1(B) shows a schematic of the binding of AA to CY2J2's active site. Figure 1(C) shows a schematic of a single cup with a low concentration of CYP2J2-ND binding on the nanoLCA surface. The morphology of the nanoLCA device was confirmed by AFM as shown in Figure 1(D). Overall, the sensor top surface has uniform hole sizes with few defects. After metal deposition, the nanohole diameter is $180 \mathrm{~nm}$ and the periodicity of the holes (distance between two neighboring holes) is $350 \mathrm{~nm}$ as measured from SEM images and AFM line scans as shown in Figure S1 and S2. 
The type of binding and dissociation constants of the substrates with CYP2J2-ND were determined by solution-based absorption spectroscopy titration assays. The binding titration data of TFN and DAN with CYP2J2-ND is shown in Figure 2(A) and 2(B), respectively. The absorbance difference spectra were measured at varying concentrations of the two drugs. The difference between the peaks and troughs of the absorbance difference spectra were then plotted for the increasing concentrations. The dissociation constants for the two drugs were then determined by fitting the plot to the Michaelis-Menten equation given as $A=\frac{A_{\max }[S]}{K_{S}+[S]}$, where $A$ is the absorbance, $A_{\max }$ is the maximum absorbance, $[S]$ is the concentration of the substrate, and $K_{S}$ is the spin state constant. For P450s, the dissociation constant, $K_{D}$, is approximated by $K_{S}$ as determined by measuring the absorbance shift as substrates bind near the heme and change the spin state of the iron from low to high spin states in the case of type I binding, resulting in a blue shift in the Soret at $417 \mathrm{~nm}$. Substrates that coordinate the iron of the heme are termed type II substrates and produce characteristic red shifts of Soret at $417 \mathrm{~nm}$. By titration assays, the dissociation constants of the substrates with CYP2J2-ND were found to be $20 \mu \mathrm{M}$ for AA, $18 \mu \mathrm{M}$ for $2-\mathrm{AG}, 12 \mu \mathrm{M}$ for ebastine, $10.8 \mu \mathrm{M}$ for $\mathrm{MSPPOH}, 5.6 \mu \mathrm{M}$ for TFN, and $1.44 \mu \mathrm{M}$ for DAN. The dissociation constants for AA, 2-AG, ebastine, and MSPPOH with CYP2J2-ND matches well with previous results (McDougle et al., 2013; McDougle et al., 2014). Due to the high absorption of DOX around $417 \mathrm{~nm}$, DOX's signal occludes the heme absorption and the dissociation constant of DOX with CYP2J2-ND cannot be determined by this conventional titration assay.

\subsection{Substrate Response Curves and CYP2J2-Nanodisc Surface Coverage on NanoLCA}

Sensor. Given these experimental values for the dissociations constants, we determined the response curve for the number of molecules on the sensor surface as a function of concentration 
of the substrate in solution, as shown in Figure 3. As can be seen, despite the fact that the dissociation constants for the six measured substrates are the same order of magnitude, there is a detectable difference of the response of CYP2J2-NDs at a wide range of substrate concentrations. The separation between two molecules $(d)$ at a certain concentration was calculated a $d=1.18 C^{-\frac{1}{3}}$, where $d$ is in nm and $C$ is in M. At a concentration of $\sim \mu \mathrm{M}$, the distance between two CYP2J2-NDs will be $\sim 118 \mathrm{~nm}$. Therefore, in $1 \mu \mathrm{M}^{2}$ area we can fit about 81 CYP2J2-NDs (assuming a mass of 320kDa and a minimum diameter of $9 \mathrm{~nm}$ for each CYP2J2-ND). The maximum coverage on our sensor is calculated to be $\Gamma_{\max }=383 \mathrm{pgcm}^{-2}$, which corresponds to a monolayer of protein covering approximately $50 \%$ of the surface. Details of the calculations performed to determine the separation between molecules, CYP2J2-ND molecular weight, and height of CYP2J2-ND/MUA system are given in the supplementary information.

The binding and unbinding interactions between immobilized CYP2J2-ND and the target drug were modeled with the following equation:

$d[C Y P 2 J 2-D r u g] / d t=k_{\text {on }}[C Y P 2 J 2][$ Drug $]-k_{\text {off }}[C Y P 2 J 2-D r u g]$

where $k_{\text {on }}$ and $k_{\text {off }}$ are the reaction coefficients for binding and release of substrate molecules, respectively. In terms of surface coverage equation (2) can be written as:

$\partial \Gamma(t) / \partial t=k_{o n} C\left[\Gamma_{\max }-\Gamma(t)\right]-k_{o f f} \Gamma(t)$

where $\Gamma_{\max }$ is the surface coverage when no more sites are available for substrate binding and $C$ is the concentration of the substrate. Note that $k_{\text {on }}$ and $k_{\text {off }}$ have different units: $\mathrm{M}^{-1} \mathrm{~s}^{-1}$ for $k_{\text {on }}$ and $\mathrm{s}^{-1}$ for $k_{\text {off }}$.

Solving equation (3) with the boundary condition $\Gamma(0)=0$ we obtain 
$\Gamma(t)=\frac{k_{o n} C \Gamma_{\max }}{k_{\text {on }} C+k_{\text {off }}}\left[1-\exp \left\{-\left(k_{o n} C+k_{\text {off }}\right) t\right\}\right]$.

At equilibrium the rates of binding and release are equal. Hence, with the condition,

$\Gamma(t \rightarrow \infty)=\Gamma_{e q}$

equation (3) can be written as:

$\frac{\Gamma_{e q}}{\Gamma_{\max }}=\frac{C}{C+K_{D}}$

where $K_{D}$ is the dissociation constant $\left(K_{D}=k_{o f f} / k_{o n}\right)$ approximated as $K_{S}$ for P450s.

3.3 CYP2J2-Nanodisc Immobilization and NanoLCA Reliability. A representative red shift in the transmission peak for a single well corresponding to immersion of the nanoLCA sensor in MUA and the CYP2J2-ND/EDC solution is shown in Figure 5(A). In order to assess the overall success of immobilization and the reliability of the nanoLCA sensor, the average and standard deviation of the plasmon resonance peak wavelength in air was determined for all wells used in this study. The averages and standard deviations of the shifts after MUA and CYP2J2-ND/EDC immersion were also determined. The raw transmission spectra in air for the nanoLCA wells is given in Figure S5. While between wells there is a variation in intensity, the centroids of the peaks are consistent. The plasmon resonance wavelength in air was $538 \pm 2 \mathrm{~nm}$, as shown in Figure 5(B), with a FWHM of $69 \pm 3 \mathrm{~nm}$. Within a single device, the standard deviation of the peak position and FWHM decreases even further as shown in Figure S6. The shift following MUA immobilization was $2 \pm 4 \mathrm{~nm}$ and the shift from air following CYP2J2-ND binding was $5 \pm 2 \mathrm{~nm}$. The shift for the vehicle control, containing only the $10 \mathrm{mM}$ KPi buffer, never exceeded $1 \mathrm{~nm}$. SEM images and a MATLAB program was used to examine variation of a single sensor on 
the microscale. SEM images used for the analysis along with histograms of hole diameter and periodicity are shown in Figures S7-S11.

\subsection{FDTD Simulation of Substrate Binding to Cytochrome P450 in Nanodiscs on the}

NanoLCA sensor. In order to predict the plasmonic peak shift of P450s on the nanoLCA sensor following substrate binding, FDTD was used to simulate three different conditions, i.e. P450 binding with (1) no substrate, (2) with type I substrate and (3) with type II substrate. The real part of refractive index was calculated from Kramers-Kronig relations for all three cases. The imaginary parts of these three cases, which corresponds to absorption coefficient, are based on measured data and shown in Figure 4(A). The measured absorbance spectra and the simulated transmission spectra of all three cases are shown in Figure 4(B) and (C) respectively. It can be observed that the resonance peak at 465 and $605 \mathrm{~nm}$ of P450 with no drug binding underwent a blue shift to 462 and $598 \mathrm{~nm}$ with the binding of a type I substrate. On the other hand, when a type II substrate was applied to bind on the surface of the nanoLCA, these two resonance peaks were red shifted to 472 and $608 \mathrm{~nm}$. These shifts agrees with previous results (Das et al., 2009). Figure 4(D-F) shows the calculated electric field distribution in z-direction for P450, P450 with type I substrate, and P450 with type II substrate, respectively. The electric field for P450 without substrate and with type II substrate are mostly dipolar in nature, whereas for type I substrate, the electric field is quadrupole in nature (Figure S12). Due to strong charge accumulation on top side of the nanohole rim (Figure 4(E)), the effective dipole moment of the system is increased for the type I substrate case compared to without substrate and with the type II substrate case. The increased dipole moment induces a higher restoring force for the plasmons, which leads to a shift of the plasmon resonance to higher energy (blue shift in the resonance wavelength). 
3.5 Spectral Shifts of CYP2J2-Nanodiscs after Substrate Binding. Table 1 gives a summary of the seven substrates tested for their solution-based and surface-based binding with CYP2J2NDs. From the solution-based absorbance data, it was determined that all seven substrates (AA, 2-AG, ebastine, MSPPOH, TFN, DAN, and DOX) result in a blue shift in the Soret band of CYP2J2-ND and therefore are classified as type I substrates for this cytochrome $\mathrm{P} 450$ protein. As expected for type I substrates, the spectral shift of immobilized CYP2J2-ND on the nanoLCA sensor following substrate binding was consistently to lower wavelengths (blue shift). Despite the fact that the dissociation constants of the seven substrates used here are similar, substratedependent shifts were measured in addition to concentration-dependent blue shifts. Figure 5(C) shows a representative blue shift in the transmission spectrum plasmon resonance peak of CYP2J2-NDs immobilized on the nanoLCA sensor following immersion in a solution containing a high concentration of ebastine. For comparison, Figure 5(D) and Figure 5(E) show the shift after immersion in a low concentration of ebastine and the $10 \mathrm{mM}$ KPi buffer, respectively. The lower concentration of the substrate leads to a lower magnitude spectral blue shift on the nanoLCA and incubation in the KPi buffer alone does not lead to a detectable shift. Additional transmission spectra are shown for AA, 2-AG, and MSPPOH in Figure S13. Figure 5(F) gives a bar graph summary of the blue shifts corresponding to substrate binding to CYP2J2-NDs on the nanoLCA sensor for all seven substrates at high and low concentrations.

3.6 Colorimetric Detection of Substrate Binding to CYP2J2-Nanodiscs. The nanoLCA sensor has a single transmission peak in the visible range and therefore bright-field microscope images can be used to detect spectral shifts following substrate binding. In order to carry out the image analysis, the original image of $2048 \times 2048$ pixels with 24 bit depth was first converted to an 8 bit image by separating the red, green, and blue channels in ImageJ software. Figure 6(A) shows the 
intensity of the green channel for MUA, CYP2J2-ND, and ebastine binding in sequence on the nanoLCA false colored in the intensity ranges of $0-255$. The average intensity of the red, green, and blue color channels for MUA, CYP2J2-ND, and ebastine is shown in Figure 6(B). All three color channels follow a similar pattern showing an overall intensity increase. Figure 6(C) shows the intensity percentage, calculated as the average intensity of each color channel divided by the sum of the average intensity for each color channel. It can be seen that from MUA to CYP2J2ND there is a detectable increase in the red and green intensity percentages and a detectable decrease in the blue intensity percentage between the images. These changes in the intensity percentages of the red, green, and blue color channels match what would be predicted for a spectral red shift on the nanoLCA device, corresponding to the spectral data we collected for CYP2J2-ND binding to immobilized MUA. From CYP2J2-ND to ebastine, there is a detectable decrease in the red intensity percentage and a detectable increase in the green intensity percentage. For comparison, the human eye has three cone visual pigments for conveying color information that can detect approximately 10 million unique colors (Sharma et al., 1997). The changes in the color channel intensity percentages indicate that a blue shift is occurring on the nanoLCA device, confirming the spectral data we collected for ebastine binding to CYP2J2-ND. The potential use of the nanoLCA sensor for detecting drug binding to P450s by image analysis alone could drastically improve assay speed allowing high-throughput screening of hundreds of drugs binding to P450s simultaneously.

\subsection{Discussion of Detection of Substrate Binding to CYP2J2-Nanodiscs on NanoLCA.}

Overall, these results suggest that the nanoLCA sensor allows spectral and colorimetric analysis of substrate binding to CYP2J2-NDs that can be carried out using a bright-field microscope, camera, and portable spectrometer. The development of a platform for high-throughput screening 
of drug binding to P450s will require a device with a small footprint, low cost, sensitive detection, and that can allow parallel detection of hundreds of different drugs at different concentrations. Nanoplasmonic sensors are an excellent candidate since they can be easily miniaturized. In particular, nanohole sensors only require a light source and portable spectrometer for data acquisition. The spectral reliability of the nanoLCA sensor that we have reported here potentially allows quantitative detection of the binding of different substrates at different concentrations to P450s. We have shown that our method can be used to detect different substrates of the same binding type. A FDTD simulation study confirmed the spectral blue shift of type I binding on the nanoLCA device. In addition, we have also presented the potential to use the nanoLCA device to perform colorimetric detection of substrate binding to CYP2J2-NDs due to its distinct spectral properties. This technology should also be reusable with substrates that bind in equilibrium, as it would only require washing with buffer to unbind them.

\section{Conclusion}

Here we have reported the application of a nanoLCA sensor to the detection of substrate binding to CYP2J2-NDs. The nanoLCA fabrication method relies on wafer-scale nanoreplica molding, which results in uniform sensors with minimal defects. We found the spectral properties of the nanoLCA sensor to be highly reliable, which allowed the detection of substrate dependent and concentration dependent blue shifts, corresponding to the binding of type I substrates to CYP2J2NDs. Our results were confirmed by traditional solution-based absorption spectroscopy and a FDTD simulation study. We also demonstrated the ability to use brightfield microscope images alone to extract spectral information, based on the fact that the nanoLCA spectrum consists of a single transmission peak in the visible. This study indicates that the nanoLCA sensor has a strong 
potential for the future development of a high-throughput spectroscopic on-chip method for detecting drug binding to cytochrome P450 proteins.

\section{Author Contributions}

LP and MRG fabricated sensors, did the experiments, analyzed the data and wrote the manuscript. WRA prepared the protein samples, performed the titration measurements, analyzed the data and wrote the paper, AA performed the microfluidics and did the data analysis, TWC did the simulation studies and wrote the paper, AH did the preliminary experiments, GGL and AD supervised the research and wrote the manuscript.

\section{Acknowledgements}

The authors thank Snehita Sri Varma for the preparation of P450 protocol and help in the initial experiments. We thank Daniel McDougle for helpful discussions. We also want to thank Sligar lab for providing the clone for membrane scaffold protein. We thank Prof. Bagchi and Prof. Ferguson for use of their equipment. The authors also thank Xiangfei Zhou for the schematic figures. We thank Hoang Nguyen, Cindy Larson, and Tiziana Bond of Lawrence Livermore National Laboratory for preparation of the master nanopillar mold. We thank National Science Foundation IGERT 0903622 for funding Lisa Plucinski. We want to thank American Heart Association for funding Aditi Das [15SDG25760064]. 


\section{References}

Anker, J.N., Hall, W.P., Lyandres, O., Shah, N.C., Zhao, J., Van Duyne, R.P., 2008. Biosensing with plasmonic nanosensors. Nat Mater 7(6), 442-453.

Baciu, C.L., Becker, J., Janshoff, A., Sonnichsen, C., 2008. Protein-membrane interaction probed by single plasmonic nanoparticles. Nano Lett 8(6), 1724-1728.

Bayburt, T.H., Sligar, S.G., 2002. Single-molecule height measurements on microsomal cytochrome P450 in nanometer-scale phospholipid bilayer disks. Proc Natl Acad Sci U S A 99(10), 6725-6730.

Bayburt, T.H., Sligar, S.G., 2003. Self-assembly of single integral membrane proteins into soluble nanoscale phospholipid bilayers. Protein Sci 12(11), 2476-2481.

Das, A., Zhao, J., Schatz, G.C., Sligar, S.G., Van Duyne, R.P., 2009. Screening of type I and II drug binding to human cytochrome P450-3A4 in nanodiscs by localized surface plasmon resonance spectroscopy. Anal Chem 81(10), 3754-3759.

Davydov, D.R., Halpert, J.R., 2008. Allosteric P450 mechanisms: multiple binding sites, multiple conformers or both? Expert Opin Drug Metab Toxicol 4(12), 1523-1535.

Delozier, T.C., Kissling, G.E., Coulter, S.J., Dai, D., Foley, J.F., Bradbury, J.A., Murphy, E., Steenbergen, C., Zeldin, D.C., Goldstein, J.A., 2007. Detection of human CYP2C8, CYP2C9, and CYP2J2 in cardiovascular tissues. Drug Metab Dispos 35(4), 682-688.

Denisov, I.G., Grinkova, Y.V., Lazarides, A.A., Sligar, S.G., 2004. Directed self-assembly of monodisperse phospholipid bilayer Nanodiscs with controlled size. J Am Chem Soc 126(11), 3477-3487.

Escobedo, C., 2013. On-chip nanohole array based sensing: a review. Lab Chip 13(13), 24452463.

Ganesh, N., Zhang, W., Mathias, P.C., Chow, E., Soares, J.A., Malyarchuk, V., Smith, A.D., Cunningham, B.T., 2007. Enhanced fluorescence emission from quantum dots on a photonic crystal surface. Nat Nanotechnol 2(8), 515-520.

Gartia, M.R., Hsiao, A., Pokhriyal, A., Seo, S., Kulsharova, G., Cunningham, B.T., Bond, T.C., Liu, G.L., 2013. Colorimetric Plasmon Resonance Imaging Using Nano Lycurgus Cup Arrays. Advanced Optical Materials 1(1), 68-76.

Gillam, E.M., Baba, T., Kim, B.R., Ohmori, S., Guengerich, F.P., 1993. Expression of modified human cytochrome P450 3A4 in Escherichia coli and purification and reconstitution of the enzyme. Arch Biochem Biophys 305(1), 123-131.

Gordon, R., Sinton, D., Kavanagh, K.L., Brolo, A.G., 2008. A new generation of sensors based on extraordinary optical transmission. Acc Chem Res 41(8), 1049-1057.

Guengerich, F.P., 1999. Cytochrome P-450 3A4: regulation and role in drug metabolism. Annu Rev Pharmacol Toxicol 39, 1-17.

Guengerich, F.P., 2008. Cytochrome p450 and chemical toxicology. Chem Res Toxicol 21(1), 70-83.

Haes, A.J., Van Duyne, R.P., 2004. A unified view of propagating and localized surface plasmon resonance biosensors. Anal Bioanal Chem 379(7-8), 920-930.

Haes, A.J., Zou, S., Zhao, J., Schatz, G.C., Van Duyne, R.P., 2006. Localized surface plasmon resonance spectroscopy near molecular resonances. J Am Chem Soc 128(33), 10905-10914.

Hashizume, T., Imaoka, S., Mise, M., Terauchi, Y., Fujii, T., Miyazaki, H., Kamataki, T., Funae, Y., 2002. Involvement of CYP2J2 and CYP4F12 in the metabolism of ebastine in human intestinal microsomes. J Pharmacol Exp Ther 300(1), 298-304. 
Hsiao, A., Gartia, M.R., Chang, T.-W., Wang, X., Khumwan, P., Liu, G.L., 2015. Colorimetric plasmon resonance microfluidics on nanohole array sensors. Sensing and Bio-Sensing Research $5(0), 24-32$.

Im, H., Shao, H., Park, Y.I., Peterson, V.M., Castro, C.M., Weissleder, R., Lee, H., 2014. Labelfree detection and molecular profiling of exosomes with a nano-plasmonic sensor. Nat Biotech 32(5), 490-495.

Iqbal, M., Gleeson, M.A., Spaugh, B., Tybor, F., Gunn, W.G., Hochberg, M., Baehr-Jones, T., Bailey, R.C., Gunn, L.C., 2010. Label-Free Biosensor Arrays Based on Silicon Ring Resonators and High-Speed Optical Scanning Instrumentation. Selected Topics in Quantum Electronics, IEEE Journal of 16(3), 654-661.

Isin, E.M., Guengerich, F.P., 2006. Kinetics and thermodynamics of ligand binding by cytochrome P450 3A4. J Biol Chem 281(14), 9127-9136.

Isin, E.M., Guengerich, F.P., 2008. Substrate binding to cytochromes P450. Anal Bioanal Chem 392(6), 1019-1030.

Ji, J., Yang, J.C., Larson, D.N., 2009. Nanohole arrays of mixed designs and microwriting for simultaneous and multiple protein binding studies. Biosens Bioelectron 24(9), 2847-2852.

Karlsson, R., Stahlberg, R., 1995. Surface plasmon resonance detection and multispot sensing for direct monitoring of interactions involving low-molecular-weight analytes and for determination of low affinities. Anal Biochem 228(2), 274-280.

Lafite, P., Andre, F., Zeldin, D.C., Dansette, P.M., Mansuy, D., 2007. Unusual regioselectivity and active site topology of human cytochrome P450 2J2. Biochemistry 46(36), 10237-10247.

Lee, C.A., Neul, D., Clouser-Roche, A., Dalvie, D., Wester, M.R., Jiang, Y., Jones, J.P., 3rd, Freiwald, S., Zientek, M., Totah, R.A., 2010. Identification of novel substrates for human cytochrome P450 2J2. Drug Metab Dispos 38(2), 347-356.

Lin, V.S., Motesharei, K., Dancil, K.P., Sailor, M.J., Ghadiri, M.R., 1997. A porous siliconbased optical interferometric biosensor. Science 278(5339), 840-843.

Luthra, A., Denisov, I.G., Sligar, S.G., 2011. Spectroscopic features of cytochrome P450 reaction intermediates. Arch Biochem Biophys 507(1), 26-35.

McDougle, D.R., Kambalyal, A., Meling, D.D., Das, A., 2014. Endocannabinoids anandamide and 2-arachidonoylglycerol are substrates for human CYP2J2 epoxygenase. J Pharmacol Exp Ther 351(3), 616-627.

McDougle, D.R., Palaria, A., Magnetta, E., Meling, D.D., Das, A., 2013. Functional studies of N-terminally modified CYP2J2 epoxygenase in model lipid bilayers. Protein Sci 22(7), 964-979.

Nath, A., Atkins, W.M., Sligar, S.G., 2007. Applications of phospholipid bilayer nanodiscs in the study of membranes and membrane proteins. Biochemistry 46(8), 2059-2069.

Piliarik, M., Vaisocherova, H., Homola, J., 2009. Surface plasmon resonance biosensing. Methods Mol Biol 503, 65-88.

Rich, R.L., Myszka, D.G., 2000. Advances in surface plasmon resonance biosensor analysis. Curr Opin Biotechnol 11(1), 54-61.

Rittle, J., Green, M.T., 2010. Cytochrome P450 compound I: capture, characterization, and C-H bond activation kinetics. Science 330(6006), 933-937.

Sligar, S.G., 2003. Finding a single-molecule solution for membrane proteins. Biochem Biophys Res Commun 312(1), 115-119.

Spector, A.A., Fang, X., Snyder, G.D., Weintraub, N.L., 2004. Epoxyeicosatrienoic acids (EETs): metabolism and biochemical function. Prog Lipid Res 43(1), 55-90. 
Wells, A.V., Li, P., Champion, P.M., Martinis, S.A., Sligar, S.G., 1992. Resonance Raman investigations of Escherichia coli-expressed Pseudomonas putida cytochrome P450 and P420. Biochemistry 31(18), 4384-4393.

Whitlock, J., Jr., Denison, M., 1995. Induction of Cytochrome P450 Enzymes That Metabolize Xenobiotics. In: de Montellano, P.O. (Ed.), Cytochrome P450, pp. 367-390. Springer US.

Wu, H.J., Henzie, J., Lin, W.C., Rhodes, C., Li, Z., Sartorel, E., Thorner, J., Yang, P., Groves, J.T., 2012. Membrane-protein binding measured with solution-phase plasmonic nanocube sensors. Nat Methods 9(12), 1189-1191.

Wu, S., Moomaw, C.R., Tomer, K.B., Falck, J.R., Zeldin, D.C., 1996. Molecular cloning and expression of CYP2J2, a human cytochrome P450 arachidonic acid epoxygenase highly expressed in heart. J Biol Chem 271(7), 3460-3468.

Yanik, A.A., Huang, M., Kamohara, O., Artar, A., Geisbert, T.W., Connor, J.H., Altug, H., 2010. An optofluidic nanoplasmonic biosensor for direct detection of live viruses from biological media. Nano Lett 10(12), 4962-4969.

Zelasko, S., Palaria, A., Das, A., 2013. Optimizations to achieve high-level expression of cytochrome P450 proteins using Escherichia coli expression systems. Protein Expression and Purification 92(1), 77-87.

Zeldin, D.C., 2001. Epoxygenase Pathways of Arachidonic Acid Metabolism. Journal of Biological Chemistry 276(39), 36059-36062.

Zeng, S., Yong, K.-T., Roy, I., Dinh, X.-Q., Yu, X., Luan, F., 2011. A Review on Functionalized Gold Nanoparticles for Biosensing Applications. Plasmonics 6(3), 491-506.

Zhang, Y., El-Sikhry, H., Chaudhary, K.R., Batchu, S.N., Shayeganpour, A., Jukar, T.O., Bradbury, J.A., Graves, J.P., DeGraff, L.M., Myers, P., Rouse, D.C., Foley, J., Nyska, A., Zeldin, D.C., Seubert, J.M., 2009. Overexpression of CYP2J2 provides protection against doxorubicin-induced cardiotoxicity. Am J Physiol Heart Circ Physiol 297(1), H37-46.

Zhao, J., Das, A., Schatz, G.C., Sligar, S.G., Van Duyne, R.P., 2008. Resonance Localized Surface Plasmon Spectroscopy: Sensing Substrate and Inhibitor Binding to Cytochrome P450. The Journal of Physical Chemistry C 112(34), 13084-13088.

Zhao, J., Das, A., Zhang, X., Schatz, G.C., Sligar, S.G., Van Duyne, R.P., 2006. Resonance Surface Plasmon Spectroscopy: $\square$ Low Molecular Weight Substrate Binding to Cytochrome P450. Journal of the American Chemical Society 128(34), 11004-11005. 

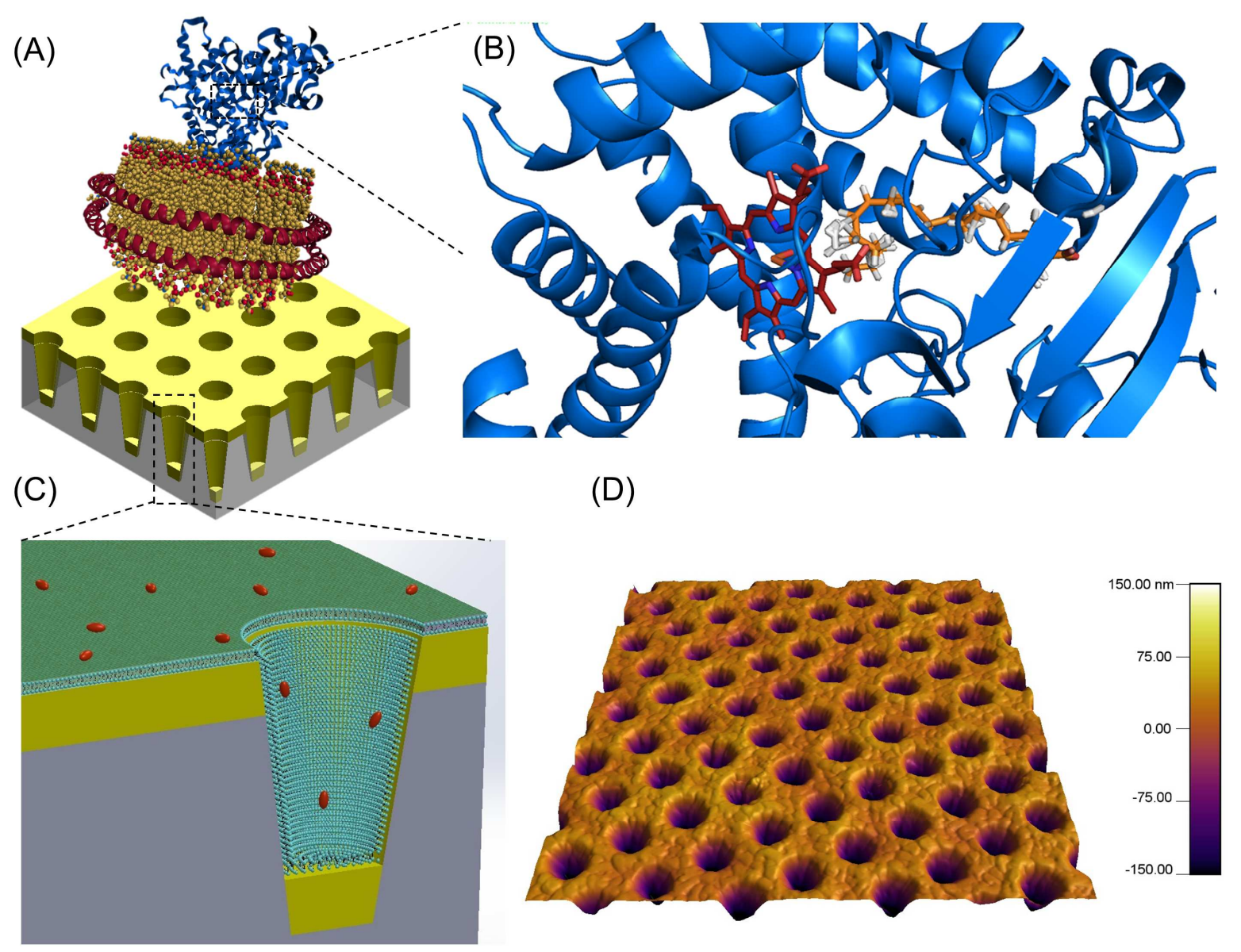

Figure 1: A schematic of the CYP2J2-ND system (A) with CYP2J2 protein (blue) incorporated into the Nanodisc is shown. The Nanodisc consists of a phospholipid bilayer (gold) and two membrane scaffold proteins (red). (B) Binding of AA to CYP2J2's active site. (C) A schematic of the cross section of a single nanocup with MUA monolayer (light blue) and CYP2J2-ND (red) bound. AFM imaging was used in order to confirm the morphology of the nanoLCA device as show in (D). 

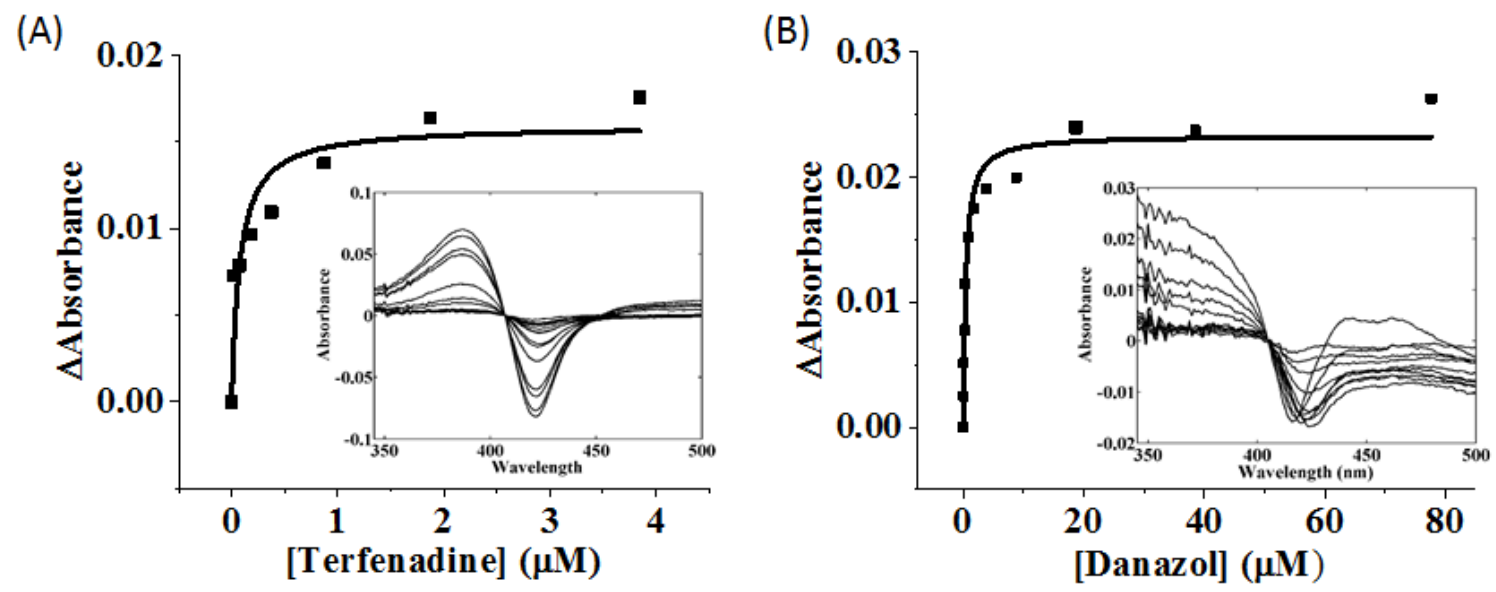

Figure 2: UV-Vis determination of TFN and DAN binding to CYP2J2. Binding was determined by measuring the heme absorbance (Soret) shift to higher wavelengths as the substrate binds and produces a higher spin state of the system. (A) TFN was titrated from 0 to $4 \mu \mathrm{M}$ and the substrate-free spectrum was subtracted from each titration (inset). The resulting differences of the peaks and troughs were then plotted and fitted to a Michaelis-Menten curve to determine a $\mathrm{K}_{\mathrm{S}}$ of $5.6 \mu \mathrm{M}$, which approximates $\mathrm{K}_{\mathrm{D}}$. (B) DAN was titrated from 0 to $80 \mu \mathrm{M}$ and the $\mathrm{K}_{\mathrm{S}}(1.44$ $\mu \mathrm{M})$ was determined similarly to (A). 


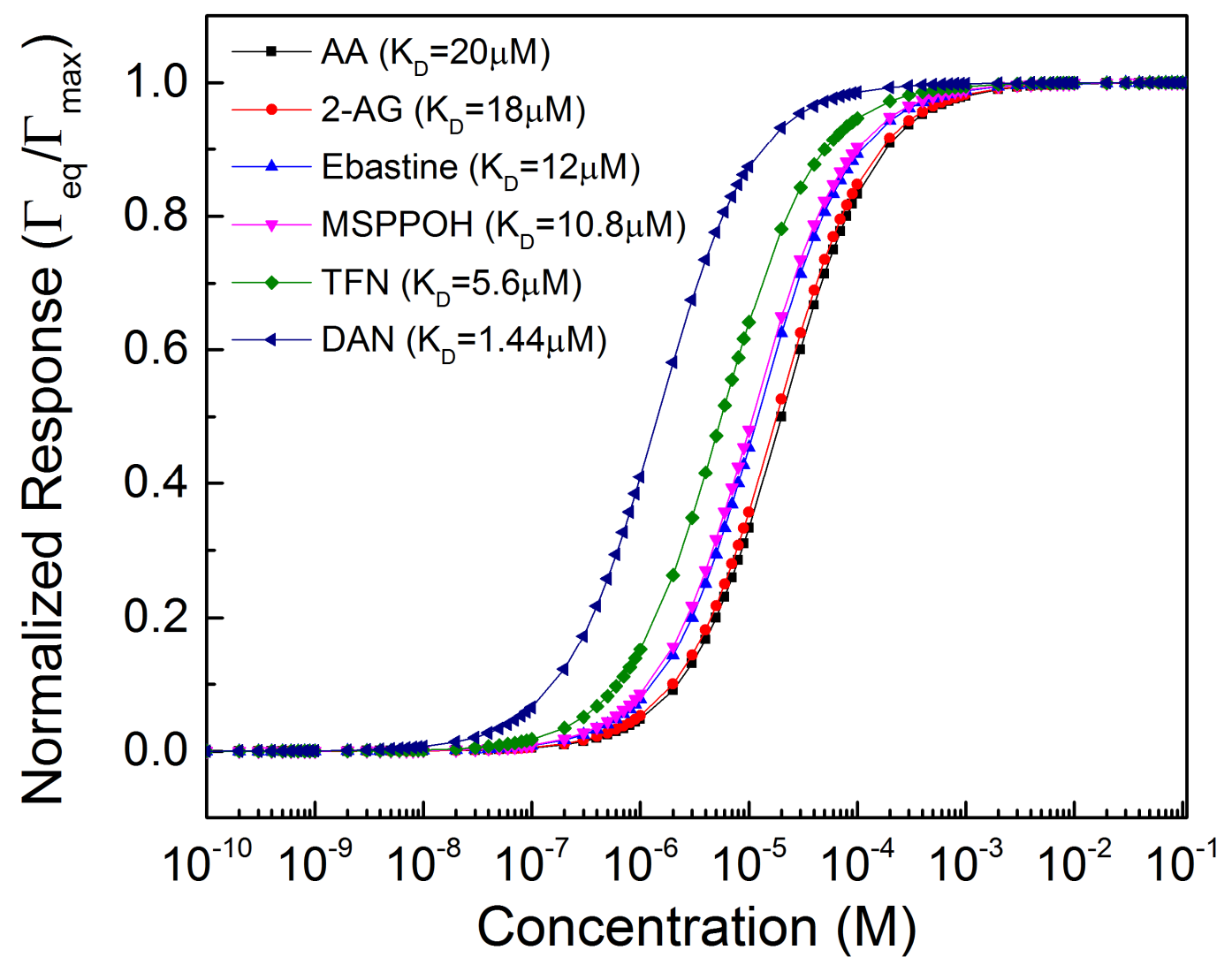

Figure 3: The response curve for number of molecules on the nanoLCA surface as a function of concentration is shown for the six different substrates with measureable dissociation constants used in this study. The curve is normalized to the maximum number of bound molecules possible. 
(A)

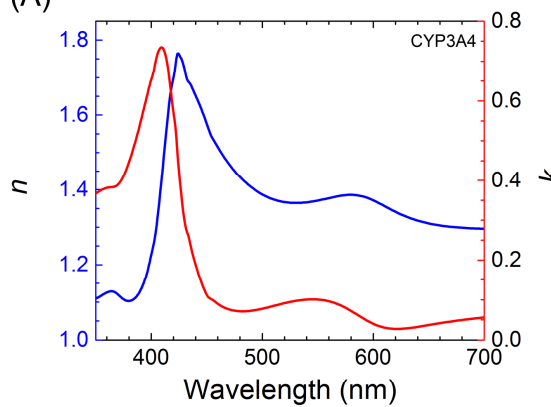

(D)

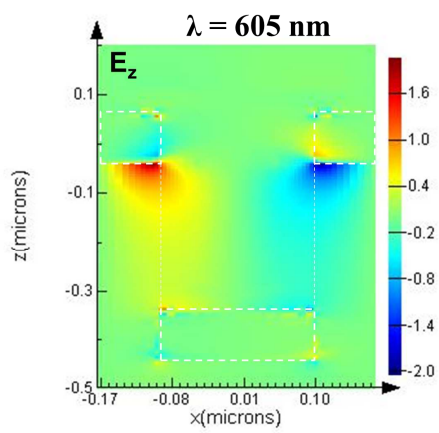

(B)

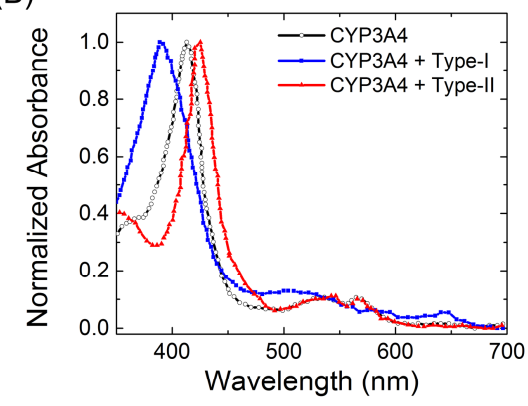

(E)

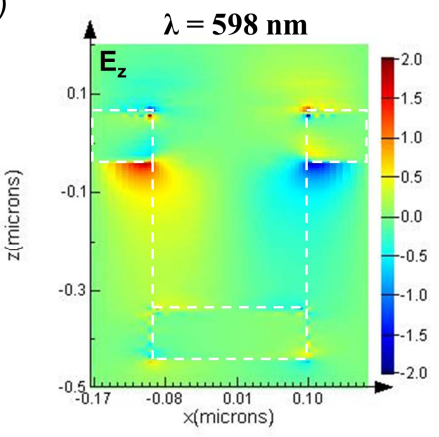

(C)

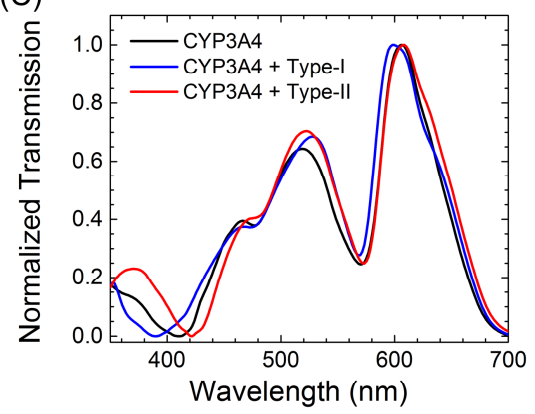

(F)

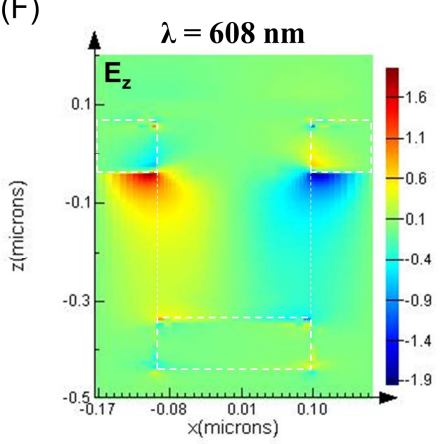

Figure 4: FDTD simulation of P-450 and substrate system (type I and type II) on top of the nanoLCA. (A) Real (n) and imaginary (k) part of the refractive index of CYP3A4 used for the electromagnetic (FDTD) simulation. (B) Normalized absorbance spectra of CYP3A4 obtained experimentally, before and after substrate binding. The black spectrum is for CYP3A4 without any substrate, the blue spectrum is for CYP3A4 with type I substrate showing blue shift of the peak resonance wavelength position, and the red spectrum is for CYP3A4 with type II substrate showing red shift of the peak resonance wavelength position. (C) Transmission spectra obtained from FDTD simulation showing CYP3A4 before (black), and after substrate binding (blue curve is for type I substrate and red curve is for type II substrate). (D-F) Calculated electric field in zdirection for CYP3A4 (D), for CYP3A4 with type I substrate (E), and for CYP3A4 with type II substrate $(\mathrm{F})$. 
(A)

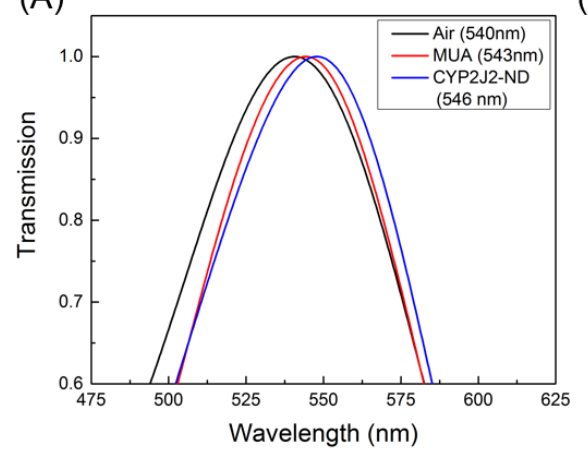

(B)

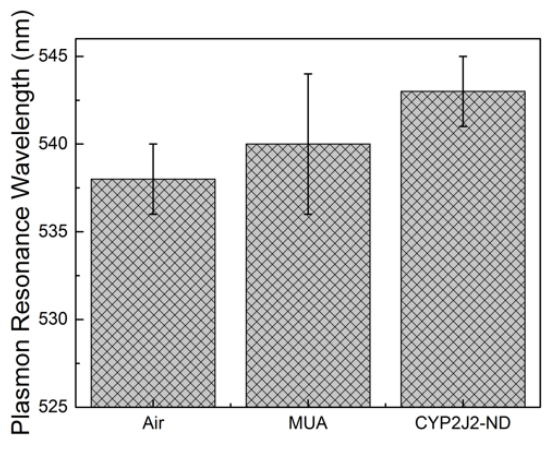

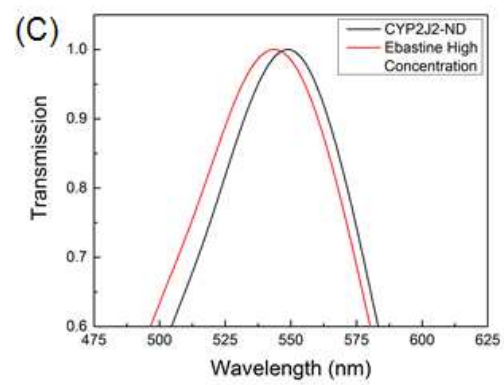
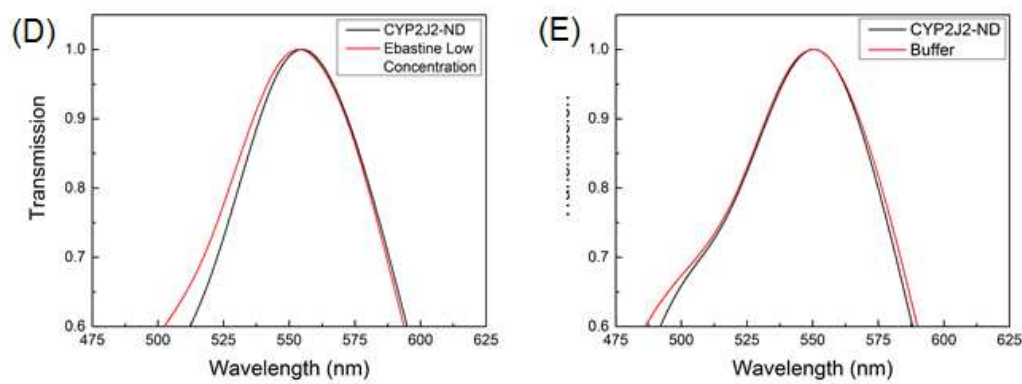

(F)

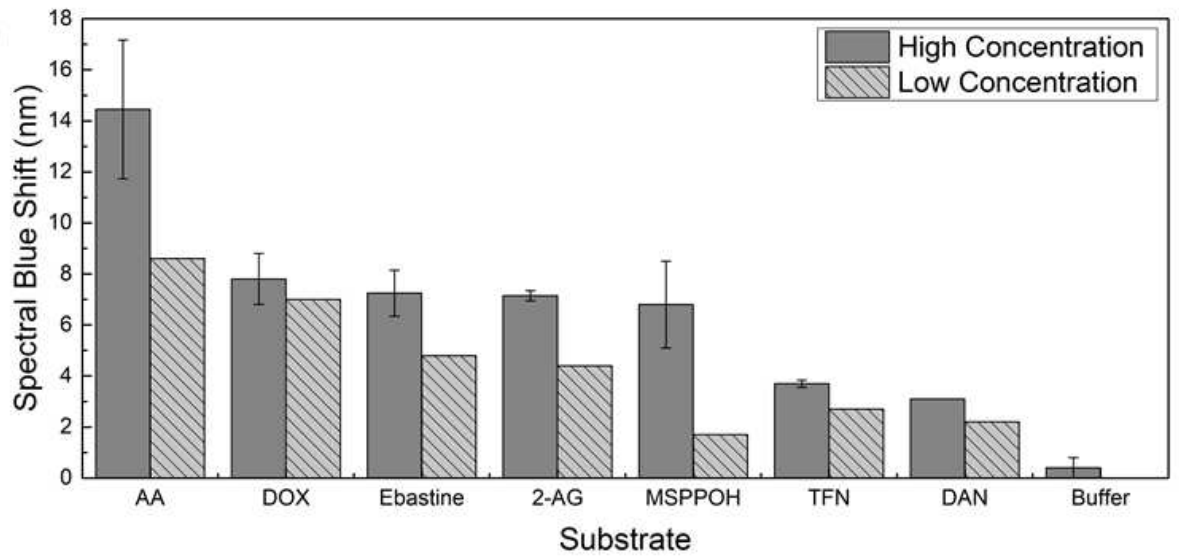

Figure 5: (A) A representative red shift in the transmission spectra following MUA

immobilization and CYP2J2-ND binding with the peak wavelength at each step noted in the figure legend. (B) A bar graph showing the average plasmon resonance peak wavelength across all wells. The spectral blue shift from a high (C) and low (D) concentration of ebastine binding with immobilized CYP2J2-ND along with the shift corresponding to 10mM KPi buffer alone (E). The lower panel bar graph shows the shifts on the nanoLCA corresponding to seven 
different type I substrates at high and low concentrations $(\mathrm{F})$. The high concentration bar corresponds to the average of the two wells with an error bar denoting the standard deviation.

(A)

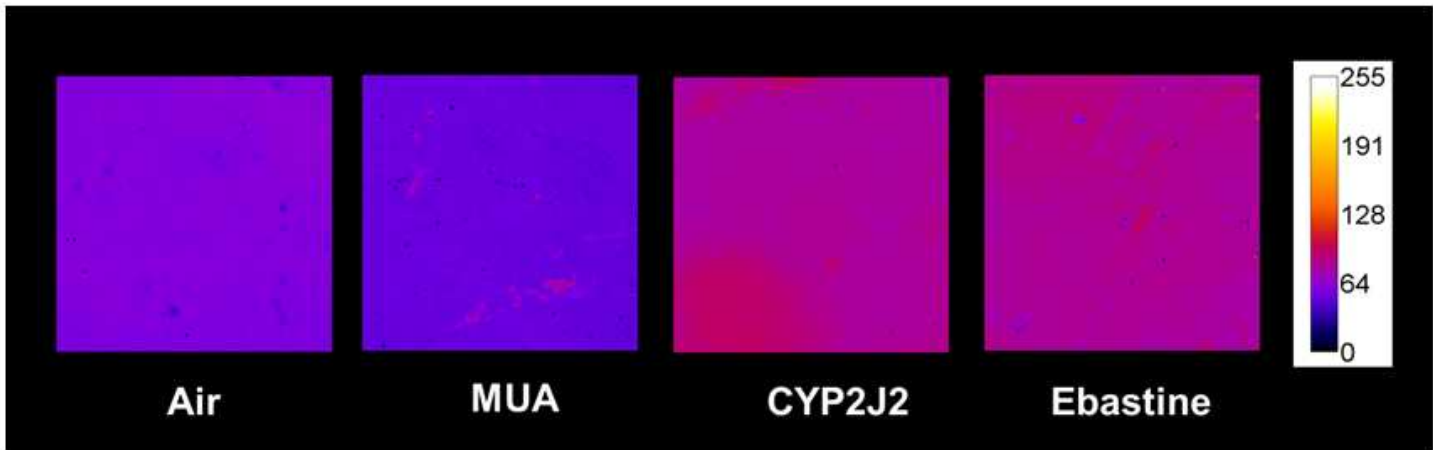

(B)

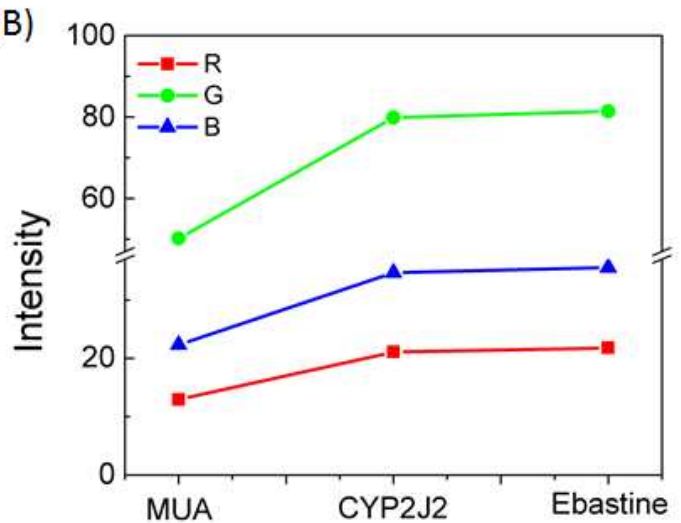

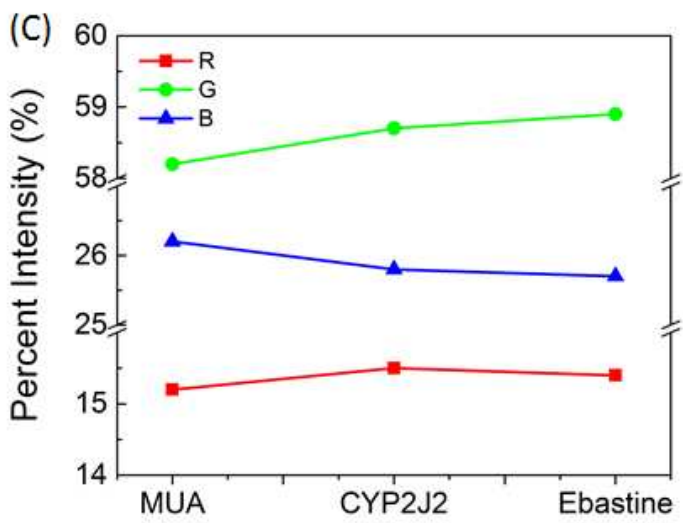

Figure 6: (A) Images of the green channel intensity for Air, MUA, CYP2J2-ND, and ebastine. The changes in average intensity for the three color channels for MUA, CYP2J2-ND, and ebastine are plotted in (B) and the percent intensity, calculated as the average intensity of the color channel divided by the total average intensity for the image, is plotted in (C). 
Table 1. Substrate Binding to CYP2J2-NDs in Solution and on NanoLCA Sensor. The binding type and dissociation constants were determined using the spectral titration shift method typical for CYPs. The direction of the plasmonic shift corroborates the binding type (Type I). Concentration-dependent plasmonic shifts are given as high and low concentrations.

\begin{tabular}{c|ccccc}
\hline Drug & Binding & Dissociation & Direction of & Plasmonic shift & Plasmonic shift \\
& type & constant $(\mu M)$ & plasmonic shift & (High conc.) & (Low conc.) \\
\hline AA & Type I & 20 & Blue & $14.5 \pm 5$ & 8.6 \\
2-AG & Type I & 18 & Blue & $7.2 \pm 0.2$ & 4.4 \\
Ebastine & Type I & 12 & Blue & $7.3 \pm 0.9$ & 4.8 \\
MSPPOH & Type I & 10.8 & Blue & $6.8 \pm 1.7$ & 1.7 \\
TFN & Type I & 5.6 & Blue & $3.7 \pm 0.1$ & 2.7 \\
DAN & Type I & 1.44 & Blue & 3.1 & 2.2 \\
DOX & Type I & Unknown & Blue & $7.8 \pm 1$ & 7 \\
\hline
\end{tabular}

